\title{
Direct measurements of black hole charge with future astrometrical missions (Research Note)
}

\author{
A. F. Zakharov ${ }^{1,2,3,4}$, F. De Paolis ${ }^{5}$, G. Ingrosso ${ }^{5}$, and A. A. Nucita ${ }^{5}$ \\ 1 National Astronomical Observatories of Chinese Academy of Sciences, 20A Datun Road, Chaoyang District, Beijing 100012, PR China \\ 2 Institute of Theoretical and Experimental Physics, 25 B. Cheremushkinskaya st., Moscow 117259, Russia \\ e-mail: zakharov@itep.ru \\ 3 Astro Space Centre of Lebedev Physics Institute, 84/32, Profsoyuznaya st., Moscow 117810, Russia \\ 4 Joint Institute for Nuclear Research, Dubna, Russia \\ 5 Department of Physics, University of Lecce and INFN, Section of Lecce, via Arnesano, 73100 Lecce, Italy
}

Received 13 May 2005 / Accepted 26 July 2005

\section{ABSTRACT}

Recently, Zakharov et al. (2005a, New Astron., 10, 479) considered the possibility of evaluating the spin parameter and the inclination angle for Kerr black holes in nearby galactic centers by using future advanced astrometrical instruments. A similar approach which uses the characteristic properties of gravitational retro-lensing images can be followed to measure the charge of a Reissner-Nordström black hole. Indeed, in spite of the fact that their formation might be problematic, charged black holes are objects of intensive investigations. From a theoretical point of view, it is well-known that a black hole is described by only three parameters, namely, its mass $M$, angular momentum $J$, and charge $Q$. Therefore, it would be important to have a method for measuring all these parameters, preferably by independent model of any. In this paper, we propose a procedure to measure the black hole charge by using the size of the retro-lensing images that can be revealed by future astrometrical missions. A discussion of the Kerr-Newmann black hole case is also offered.

Key words. black hole physics - astrometry - gravitation - gravitational lensing - galaxies: nuclei

\section{Introduction}

"Black holes have no hair" means that a black hole is characterized by only three parameters ("hairs"), its mass $M$, angular momentum $J$, and charge $Q$ (see, e.g. Misner et al. 1973; Wald 1984 or Heusler 1998 for a more recent review). Therefore, in principle, charged black holes can be formed, although astrophysical conditions that lead to their formation may look rather problematic: see, for example, Zamir (1993); Ruffini et al. (1999); Ruffini et al. (2000); Lee et al. (2001); Perjes \& Vasuth (2003); Ray et al. (2003); Moderski \& Rogatko (2004); Vogt \& Letelier (2004); Lemos \& Weinberg (2004); Sereno (2004); Ghezzi \& Letelier (2005). Nevertheless, one can not claim that their existence is forbidden by theoretical or observational arguments.

Charged black holes are also objects of intensive studies, since they are described by Reissner-Nordström geometry which is a static, spherically symmetrical solution of Yang-Mills-Einstein equations with fairly natural requirements on asymptotic behavior of the solutions (Gal'tsov \& Ershov 1988; Gal'tsov et al. 1989; Gal'tsov \& Ershov 1989; Lee et al. 1991). The Reissner-Nordström metric thus describes a spherically symmetric black hole with a color charge and (or) a magnetic monopole (see also Heusler 1998).

The formation of retro-lensing images (also known as mirage, shadows, or "faces" in the literature) due to the strong gravitational field effects near black holes has been investigated by several authors (Holz \& Wheeler 2002; De Paolis et al. 2003; De Paolis et al. 2004; Zakharov et al. 2005a,b,c). The question that naturally arises is whether these images are observable or not. It has been shown that the retro-lensing image around the black hole at the Galactic Center $\left(\operatorname{Sgr} A^{*}\right)$ due to $S_{2}$ star is observable in the $K$-band (peaked at $2.2 \mu \mathrm{m}$ ) by the next generation infra-red space-based missions. The effects of retro-lensing image shapes due to black hole spin has also been investigated (De Paolis et al. 2004; Zakharov et al. 2005a,c,e).

In this paper we focus on the possibility of measuring the black hole charge as well, and we present an analytical dependence of mirage size on the black hole charge. Indeed, future space missions like Radioastron in radio band or MAXIM in $\mathrm{X}$-ray band have angular resolution close to the shadow size for massive black holes in the center of our own and nearby galaxies. 


\section{Basic definitions and equations}

The expression for the Reissner-Nordström metric in natural units $(G=c=1)$ has the form

$$
\begin{aligned}
\mathrm{d} s^{2}=-\left(1-\frac{2 M}{r}+\frac{Q^{2}}{r^{2}}\right) \mathrm{d} t^{2}+ & \left(1-\frac{2 M}{r}+\frac{Q^{2}}{r^{2}}\right)^{-1} \mathrm{~d} r^{2} \\
& +r^{2}\left(\mathrm{~d} \theta^{2}+\sin ^{2} \theta \mathrm{d} \phi^{2}\right)
\end{aligned}
$$

Applying the Hamilton-Jacobi method to the problem of geodesics in the Reissner-Nordström metric, the motion of a test particle in the $r$-coordinate can be described by following equation (see, for example, Misner et al. 1973)

$r^{4}(\mathrm{~d} r / \mathrm{d} \lambda)^{2}=R(r)$

where

$$
\begin{aligned}
& R(r)=P^{2}(r)-\Delta\left(\mu^{2} r^{2}+L^{2}\right), \\
& P(r)=E r^{2}-e Q r \\
& \Delta=r^{2}-2 M r+Q^{2} .
\end{aligned}
$$

Here, the constants $\mu, E, L$, and $e$ are associated with the particle; i.e. $\mu$ is its mass, $E$ is energy at infinity, $L$ is its angular momentum at infinity, and $e$ is the particle's charge.

We shall consider the motion of uncharged particles $(e=0)$ below. In this case, the expression for the polynomial $R(r)$ takes the form

$$
\begin{array}{r}
R(r)=\left(E^{2}-\mu^{2}\right) r^{4}+2 M \mu r^{3}-\left(Q^{2} \mu^{2}+L^{2}\right) r^{2} \\
+2 M L^{2} r-Q^{2} L^{2}
\end{array}
$$

Depending on the multiplicities of the roots of the polynomial $R(r)$, we can have three types of motion in the $r$-coordinate (Zakharov 1986). In particular, by defining $r_{+}=1+\sqrt{1-Q^{2}}$, we have:

(1) if the polynomial $R(r)$ has no roots for $r \geq r_{+}$, a test particle is captured by the black hole;

(2) if $R(r)$ has roots and $(\partial R / \partial r)\left(r_{\max }\right) \neq 0$ with $r_{\max }>r_{+}\left(r_{\max }\right.$ is the maximal root), a particle is scattered after approaching the black hole;

(3) if $R(r)$ has a root and $R\left(r_{\max }\right)=(\partial R / \partial r)\left(r_{\max }\right)=0$, the particle now takes an infinite proper time to approach the surface $r=$ const.

If we are considering a photon $(\mu=0)$, its motion in the $r$-coordinate depends on the root multiplicity of the polynomial $\hat{R}(\hat{r})$

$\hat{R}(\hat{r})=R(r) /\left(M^{4} E^{2}\right)=\hat{r}^{4}-\xi^{2} \hat{r}^{2}+2 \xi^{2} \hat{r}-\hat{Q}^{2} \xi^{2}$,

where $\hat{r}=r / M, \xi=L /(M e)$ and $\hat{Q}=Q / M$.

One can see from Eqs. (5) and (3) that the black hole charge may substantially influence the photon motion at small radii $(r \approx 1)$, while the charge effect is almost negligible at large radial coordinates of photon trajectories $(r \gg 1)$. In the last case, we should keep in mind that the charge may cause only small corrections to photon motion.

\section{Capture cross section of photons by a Reissner-Nordström black hole}

Let us consider the problem of the capture cross section of a photon by a charged black hole. It is clear that the critical value of the impact parameter for a photon to be captured by a Reissner-Nordström black hole depends on the multiplicity root condition of the polynomial $R(r)$, i. e. the condition for a vanishing discriminant (Zakharov 1988, 1991a,b, 1994a). In particular, it was shown that the vanishing discriminant condition approach is more powerful than the procedure that excludes $r_{\max }$ from the following system

$R\left(r_{\max }\right)=0, \quad \partial R \partial r\left(r_{\max }\right)=0$,

as was done, for example, by Chandrasekhar (1983) to solve similar problems.

Introducing the notation $\xi^{2}=l, Q^{2}=q$, we obtain

$R(r)=r^{4}-l r^{2}+2 l r-q r$.

The discriminant $\Delta$ of the polynomial $R(r)$ has the form, as it was shown by Zakharov (1991a,b, 1994a):

$\Delta=16 l^{3}\left[l^{2}(1-q)+l\left(-8 q^{2}+36 q-27\right)-16 q^{3}\right]$.

The polynomial $R(r)$ thus has a multiple root if and only if

$l^{3}\left[l^{2}(1-q)+l\left(-8 q^{2}+36 q-27\right)-16 q^{3}\right]=0$.

Excluding the case $l=0$, which corresponds to a multiple root at $r=0$, we find that the polynomial $R(r)$ has a multiple root for $r \geq r_{+}$if and only if

$l^{2}(1-q)+l\left(-8 q^{2}+36 q-27\right)-16 q^{3}=0$.

If $q=0$, we obtain the well-known result for a Schwarzschild black hole (Misner et al. 1973; Wald 1984; Lightman et al. 1975), $l=27$, or $L_{\mathrm{cr}}=3 \sqrt{3}$. If $q=1$, then $l=16$, or $L_{\mathrm{cr}}=4$, which also corresponds to numerical results given by Young (1976), De Vries (2000) and Takahashi (2005).

The photon capture cross section for an extremely charged black hole turns out to be considerably smaller than the capture cross section of a Schwarzschild black hole. The critical value of the impact parameter, characterizing the capture cross section for a Reissner-Nordström black hole, is determined by the equation (Zakharov 1991a,b, 1994a)

$l=\frac{b_{1}+\sqrt{b_{1}^{2}+64 q^{3}(1-q)}}{2(1-q)}$,

where $b_{1}=8 q^{2}-36 q+27$.

Substituting Eq. (11) into the expression for the coefficients of the polynomial $R(r)$, it is easy to calculate the radius of the unstable circular photon orbit, which is the same as the minimum periastron distance. The orbit of a photon moving from infinity with the critical impact parameter, determined in accordance with Eq. (11), spirals into circular orbit.

As was explained by Zakharov et al. (2005a,b,c) this leads to the formation of shadows described by the critical value of $L_{\mathrm{cr}}$; or in other words, in the spherically symmetric case, shadows are circles with radii $L_{\mathrm{cr}}$. Therefore, by measuring the shadow size, one could evaluate the black hole charge in black hole mass units $M$. 
Table 1. The fringe sizes (in micro arcseconds) for the standard and advanced apogees $B_{\max }(350000$ and $3200000 \mathrm{~km}$, respectively).

\begin{tabular}{ccccc}
\hline \hline$B_{\max }(\mathrm{km}) \backslash \lambda(\mathrm{cm})$ & 92 & 18 & 6.2 & 1.35 \\
\hline $3.5 \times 10^{5}$ & 540 & 106 & 37 & 8 \\
\hline $3.2 \times 10^{6}$ & 59 & 12 & 4 & 0.9 \\
\hline
\end{tabular}

\section{The space RADIOASTRON interferometer}

The space-based radio telescope RADIOASTRON ${ }^{1}$ is planned to be launched within few next years ${ }^{2}$. This space-based $10-\mathrm{m}$ radio telescope will be used for space - ground VLBI observations. The measurements will have extremely high angular resolutions, namely about $1-10 \mu$ as (in particular about $8 \mu$ as at the shortest wavelength of $1.35 \mathrm{~cm}$ and a standard orbit ${ }^{3}$, and could be about $0.9 \mu$ as for the high orbit configuration at the same wavelength. Four wave bands will be used corresponding to $\lambda=1.35 \mathrm{~cm}, \lambda=6.2 \mathrm{~cm}, \lambda=18 \mathrm{~cm}, \lambda=92 \mathrm{~cm}$ (see Table 1). A detailed calculation of the high-apogee evolving orbits $\left(B_{\max }\right)$ can be done, once the exact launch time is known.

After several years of observations, it should be possible to move the spacecraft to a much higher orbit (with apogee radius about 3.2 million $\mathrm{km}$ ), by additional spacecraft maneuvering using the gravitational force of the Moon. The fringe sizes (in $\mu$ as) for the apogee of the above-mentioned orbit and for all RADIOASTRON wavelengths are given in Table 1.

By comparing Figs. 1, 2 and Table 1, one can see that there are non-negligible chances to observe such mirages around the black hole at the Galactic Center and in nearby AGNs and microquasars in the radio-band using RADIOASTRON facilities.

We also mention that this high resolution in radio band will be achieved also by Japanese VLBI project VERA (VLBI Exploration of Radio Astrometry), since the angular resolution aimed at will be at the $10 \mu$ as level (Sawad-Satoh 2000; Honma 2001). Therefore, the only problem left is to have a powerful enough radio source to illuminate a black hole in order to have retro-lensing images detectable by such radio VLBI telescopes as RADIOASTRON or VERA.

\footnotetext{
${ }^{1}$ See web-site http://www.asc.rssi.ru/radioastron/ for more information.

2 This project was proposed by the Astro Space Center (ASC) of Lebedev Physical Institute of the Russian Academy of Sciences (RAS) in collaboration with other institutions of RAS and RosAviaKosmos. Scientists from 20 countries are developing the scientific payload for the satellite by providing by ground-based support to the mission.

3 The satellite orbit will have high apogee, and its rotation period around Earth will be 9.5 days, which evolves as a result of the weak gravitational perturbations from the Moon and the Sun. The perigee has been planned to be between $10^{4}$ and $7 \times 10^{4} \mathrm{~km}$ and the apogee between 310 and 390 thousand kilometers. The basic orbit parameters will be the following: the orbital period is $P=9.5$ days, the semimajor axis is $a=189000 \mathrm{~km}$, the eccentricity is $e=0.853$, the perigee is $H=29000 \mathrm{~km}$.
}

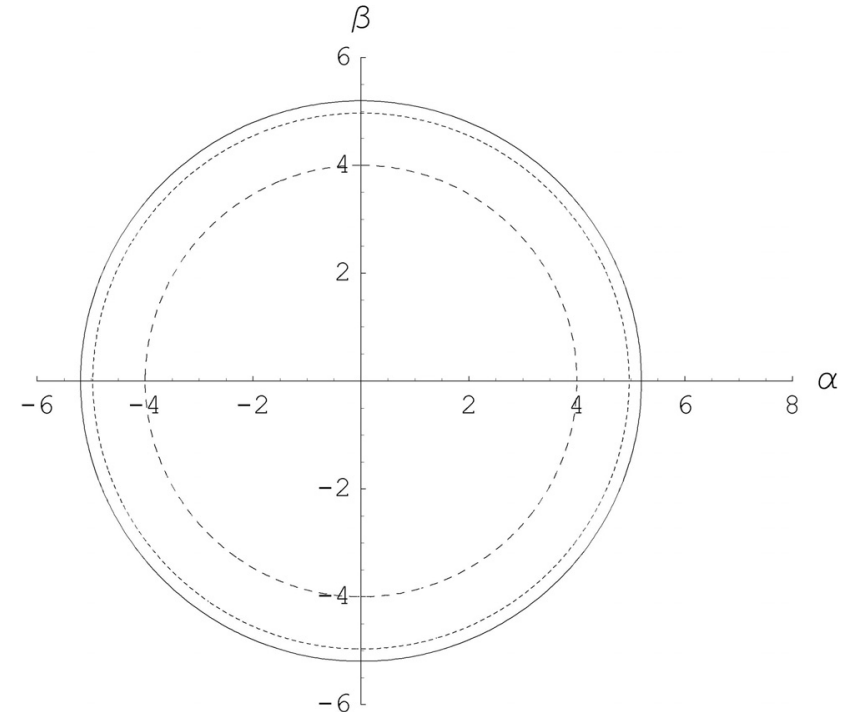

Fig. 1. Shadow (mirage) sizes are shown for selected charges of black holes $Q=0$ (solid line), $Q=0.5$ (short dashed line), and $Q=1$ (long dashed line).

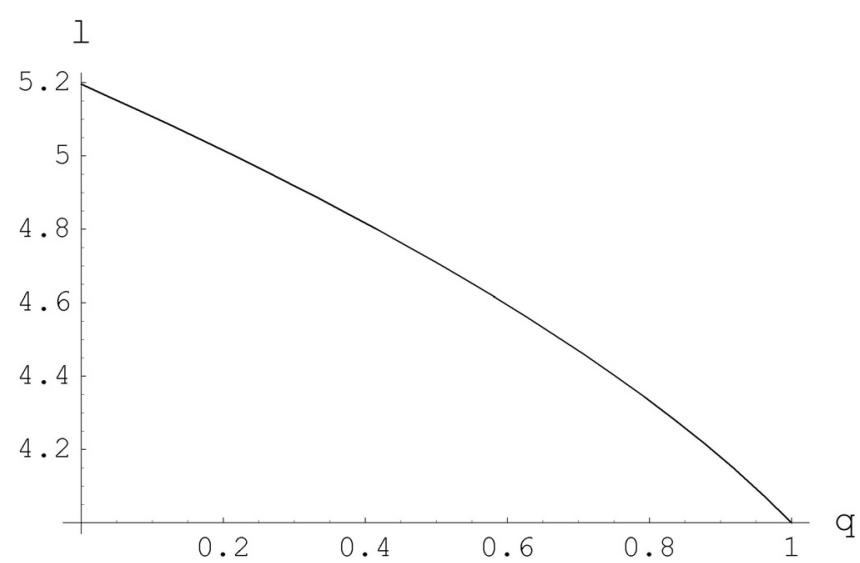

Fig. 2. The mirage radius $l$ is shown as a function of the black hole charge $q(l$ and $q$ are given in units of $M)$.

\section{Searches for mirages near Sgr $A^{*}$ with RADIOASTRON}

Radio, near-infrared, and X-ray spectral band observations are developing very rapidly (Lo et al. 1998, 1999; Genzel et al. 2003; Ghez et al. 2004; Baganoff et al. 2001, 2003; Bower et al. 2002, 2003; Narayan 2003; Bower et al. 2004) ${ }^{4}$, and it is known that $\mathrm{Sgr} A^{*}$ harbors the closest massive black hole with mass estimated to be $4.07 \times 10^{6} M_{\odot}$ (Bower et al. 2004; Melia \& Falcke 2001; Ghez et al. 2003; Schodel et al. 2003).

Following the idea of Falcke et al. (2000) and of Zakharov et al. (2005a,b,c,d) we propose to use the VLBI technique to observe mirages around massive black holes and, in particular, towards the black hole at Galactic Center. To evaluate the shadow shape Falcke et al. (2000) used the ray-tracing technique. The boundaries of the shadows are black hole mirages.

\footnotetext{
4 An interesting idea to use radio pulsars to investigate the region nearby black hole horizon was proposed recently by Pfahl \& Loeb (2003).
} 
We use the length parameter $r_{\mathrm{g}}=G M c^{2}=6 \times 10^{11} \mathrm{~cm}$ for the black hole at $\operatorname{Sgr} A^{*}$ and an analytical approach (see Sects. 2, $3)$ to calculate shadow sizes, as explained in the text. By taking the distance of $\operatorname{Sgr} A^{*}$ to be $D_{\mathrm{GC}}=8 \mathrm{kpc}$, the length $r_{\mathrm{g}}$ corresponds to angular size $\sim 5 \mu$ as. Since the minimum arc size for the considered mirages are at least $8 r_{\mathrm{g}}$, the standard RADIOASTRON resolution of about $8 \mu$ as is comparable to the required precision. The resolution in the case of the highest orbit and shortest wavelength is $\sim 1 \mu$ as (see Table 1), good enough to reconstruct the mirage shapes. As can be seen from Fig. 1 and Table 1, it is clear that, in principle, it is possible to evaluate the black hole charge $Q$ by observing the shadow size. The mirage size difference between the extreme charged black hole and Schwarzschild black hole case is about $30 \%$ (the mirage diameter for Schwarzschild black hole is about 10.4, and for the extreme charged black hole, the diameter is equal to 8 or in black hole mass units) and typical angular sizes are about $\sim 52 \mu$ as for the Schwarzschild and $\sim 40 \mu$ as for the ReissnerNordström black hole cases, respectively. Therefore, for $\operatorname{Sgr} A^{*}$ a charged black hole could be distinguished by a Schwarzschild black hole with RADIOASTRON, at least if its charge is close to the maximal value. For stellar mass black holes, we need a much higher angular resolution to distinguish charged and uncharged black holes, since the typical shadow (mirage) angular sizes are about $2 \times 10^{-5} \mu$ as, even for galactic black holes.

Actually, from the mathematical point of view we proved the following statement. For any positions of source and observer, there is an emitted photon passing close enough to any point of the mirage that can be caught by the observer. Thus, the mirage is formed by an envelope of a family of photon trajectories or, in other words, by a caustic surface.

Mirage formation under realistic assumptions for a specific case was discussed by Falcke et al. (2000). However, instead of solving the shadow formation problem by numerical simulations, one can use the approach developed by Zakharov (1994b, 1995); Zakharov \& Repin (1999, 2002a,b, 2004a, 2003b,c,d); Zakharov et al. (2004); Zakharov (2004); Zakharov \& Repin (2005a), Zakharov et al. (2005a,b). Mirages are boundaries of bright images, and shadows are also generated for accretion disks. However, for thick accretion disks as well, some parts of mirages could be observable, at least in principle.

\section{Conclusions}

The angular resolution of the space RADIOASTRON interferometer will be high enough to resolve radio images around black holes. By measuring the mirage shapes one should be able to evaluate the black hole mass, inclination angle (e.g. the angle between the black hole spin axis and line of sight), and spin, if the black hole distance is known. For example, for the black hole at the Galactic Center, the mirage size is $\sim 52 \mu$ as for the Schwarzschild case. In the case of a Kerr black hole (Zakharov et al. 2005a,b; De Paolis et al. 2004), the mirage is deformed depending on the black hole spin $a$ and on the angle of the line of sight, but its size is almost the same. In the case of a Reissner-Nordström black hole, its charge changes the size of the shadows up to $30 \%$ for the extreme charge case. Therefore, the charge of the black hole can be measured by observing the shadow size, if the other black hole parameters are known with sufficient precision. In general, one could say that a measure of the mirage shape (in size) allows to evaluate all the black hole "hairs" to be evaluated.

However, there are two kinds of difficulties when measuring mirage shapes around black holes. First, the brightness of these images or their parts (arcs) may not be sufficient for being detected by RADIOASTRON. But, numerical simulations by Falcke et al. (2000) and Melia \& Falcke (2001) give hope that the shadow brightness could be high enough to be detectable. Second, turbulent plasma effects near the black hole horizon could give essential broadening of observed images (Bower et al. 2004) leading to a confusion of the shadow image.

Recent observations of simultaneous X-ray and radio flares at $3 \mathrm{~mm}, 7 \mathrm{~mm}, 1.3 \mathrm{~cm}$, and $2 \mathrm{~cm}$ with few-hundred second rise/fall timescales gave indirect evidence that $\mathrm{X}$-ray and radio radiation from the close vicinity of $\mathrm{Sgr} \mathrm{A}^{*}$ has been detected (Baganoff et al. 2001).

A few years ago the possibility of observing the images of distant sources around black holes in the X-ray band was discussed by White (2000) and Cash et al. (2000) by using X-ray interferometer. Indeed, the aim of the MAXIM project is to realize a space-based X-ray interferometer capable of observing with angular resolution as small as $0.1 \mu \mathrm{as}$.

One could also mention that if the emitting region has a degenerate position with respect to the line of sight (for example, the inclination angle of an accretion disk is $\gtrsim 85^{\circ}$ ) strong bending effects do appear (see, Matt et al. 1993; Zakharov \& Repin 2003a).

In spite of the difficulties of measuring the shapes of shadow images, to look at black hole "faces" is an attractive challenge since mirages outline the "faces" and correspond to a fully general relativistic description of the region near the black hole horizon without any assumption about a specific model for astrophysical processes around black holes; of course, we assume that there are sources illuminating black hole surroundings. There is no doubt that the rapid growth of observational facilities will give a chance to measure the mirage shapes using not only RADIOASTRON facilities but also other instruments and spectral bands, like the X-ray interferometer MAXIM, the RADIOASTRON mission, or other space-based interferometers in millimeter and sub-millimeter bands (Miyoshi 2004).

Acknowledgements. A.F.Z. would like to thank the Dipartimento di Fisica Università di Lecce and INFN, Sezione di Lecce and National Astronomical Observatories of Chinese Academy of Sciences, Beijing, for their hospitality. A.F.Z. thanks also the National Natural Science Foundation of China (NNSFC) for partial financial support of the work (Project \#10233050).

\section{References}

Baganoff, F. K., Bautz, M. W., Brandt, W. N., et al. 2001, Nature, 413, 45

Baganoff, F. K., Maeda, Y., Morris, M., et al. 2003, ApJ, 591, 891

Bower, F. C., Falcke, H., Sault, R. J., \& Backer, D. C. 2002, ApJ, 571, 843

Bower, F. C., Wright, M. C. H., Falcke, H., \& Backer, D. C. 2003, ApJ, 588, 331 
Bower, F. C., Falcke, H., Herrstein, R. M., et al. 2004, Sciencexpress, www.sciencexpress.org/1 April 2004/

Cash, W., Shipley, A., Osterman, S., \& Joy, M. 2000, Nature, 407, 160

Chandrasekhar, S. 1983, Mathematical Theory of Black Holes (Oxford: Clarendon Press)

De Paolis, F., Geralico, A., Ingrosso, G., \& Nucita, A. A. 2003, A\&A, 409, 809

De Paolis, F., Geralico, A., Ingrosso, G., \& Nucita, A. A. 2004, A\&A, 415,1

Desai, K. M., \& Fey, A. L. 2001, ApJS, 133, 395

de Vries, A. 2000, CQG, 17, 123

Falcke, H., Melia, F., \& Agol, E. 2000, ApJ, 528, L13

Gal'tsov, D. V., \& Ershov, A. A. 1988, Yad. Fiz., 4, 460

Gal'tsov, D. V., Volkov, M. S., \& Ershov, A. A. 1989, Tr. Inst. Fiz. Akad. Nauk Est. SSR, 65, 160

Gal'tsov, D. V., \& Ershov, A. A. 1989, Phys. Lett. A, 138, 160

Genzel, R., Schödel, R., Ott, T., et al. 2003, Nature, 425, 934

Ghez, A. M., Duchêne, G., Matthews, K., et al. 2003, ApJ, 586, L127

Ghez, A. M., Wright, S. A., Matthews, K., et al. 2004, ApJ, 601, L159

Ghez, A. M., Salim, S., Hornstein, S. D., et al. 2005, ApJ, 620, 744

Ghezzi, C. R., \& Letelier, P. S. 2005 [arXiv: astro-ph/0503629]

Holz, D., \& Wheeler, J. A. 2002, ApJ, 578, 330

Honma, M. 2001, PASJ, 53, 233

Honma, M., \& Kurayama, T. 2002, ApJ, 568, 717

Heusler, M. 1998, Living Reviews in Relativity, 1998-6

Lee, H. K., Lee, C. H., \& van Putten, M. H. P. M. 2001, MNRAS, 324, 781

Lee, K., Nair, V. P., \& Weinberg, E. J. 1991, Fermilab Publ., N 91-312 Lemos, J. P. S., \& Weinberg, E. 2004, PRD, 69, 104004

Lightman, A. P., Press, W. H., Price, R. H., \& Teukolsky, S. A. 1975, Problem Book in Relativity and Gravitation (Princeton New Jersey: Princeton University Press)

Lo, K. Y., Shen, Zh.-Q., Zhao, J.-H., \& Ho, P. T. P. 1998, ApJ, 508, L61

Lo, K. Y., Shen, Zh.-Q., Zhao, J.-H., \& Ho, P. T. P. 1999, in The Central Parsecs of the Galaxy, ed. H. Falcke, A. Cotera, W. J. Duschl, F. Melia, \& M. J. Rieke, ASP Conf. Ser., 317

Matt, G., Perola, G. C., \& Stella, L. 1993, A\&A, 267, 2, 643

Melia, F., \& Falcke, H. 2001, ARA\&A, 39, 309

Misner, C. W., Thorne, K. S., \& Wheeler, J. A. 1973, Gravitation (San Francisco: Freeman)

Miyoshi, M. 2004 [arXiv: astro-ph/0412289]

Moderski, R., \& Rogatko, M. D. 2004, PRD, 69, 084018

Narayan, R. 2003, Nature, 425, 908

Perjes, Z., \& Vasuth, M. 2003, ApJ, 582, 342

Phahl, E., \& Loeb, A. 2003 [arXiv:astro-ph/0309744]

Ray, S., Espindola, A. L., Malheiro, M., et al. 2003, PRD, 68, 084004

Ruffini, R., Salmonson, J. D., Wilson, J. R., \& Xue, S.-S. 1999, A\&A, 350, 334

Ruffini, R., Salmonson, J. D., Wilson, J. R., \& Xue, S.-S. 2000, A\&A, 359,855

Schödel, R., Ott, T., Genzel, R., et al. 2003, ApJ, 596, 1015

Sawada-Sato, S. 2000, in Proc. VSOP Symp., ed. H. Hirabayashi, P. G. Edwards, \& D. W. Murphy, held at the Institute of Space and astronautical Science, Sagamihara, Kanagawa, Japan, January 19-21, 2000, the Institute of Space and Astronautical Science, 317

Sereno, M. 2004, PRD, 69, 023002

Takahashi, R. 2005 [arXiv:astro-ph/0505316]

Vogt, D., \& Letelier, P. S. 2004, [arXiv: gr-qc/0409108]

Wald, R. M. 1984, General Relativity (The Chicago University Press)
White, N. 2000, Nature, 407, 146

Wilkinson, P. N., Narayan, R., \& Spencer, R. E. 1994, MNRAS, 238, 963

Young, P. J. 1976, Phys. Rev., D, 14, 3281

Zakharov, A. F. 1986, Sov. Phys. JETP, 64, 1

Zakharov, A. F. 1988, Sov. Astron., 32, 456

Zakharov, A. F. 1991a, Sov. Astron., 35, 147

Zakharov, A. F. 1991b, Preprint ITEP, 44-91

Zakharov, A. F. 1994a, CQG, 11, 1027

Zakharov, A. F. 1994b, MNRAS, 269, 283

Zakharov, A. F. 1995, in 17th Texas Symp. Relativistic Astrophysics, Ann. NY Academy of Sciences, 759, 550

Zakharov, A. F. 2004, in The Physics of Ionized Gases, AIP Conf. Proc., ed. L. Hadzievski, T. Gvozdanov, \& N. Bibic, 740, 398 [arXiv: astro-ph/0411611]

Zakharov, A. F. 2005, Int. J. Mod. Phys. A, 20, 2321

Zakharov, A. F., Kardashev, N. S., Lukash, V. N., \& Repin, S. V. 2003, MNRAS, 342, 1325

Zakharov, A. F., Ma, Z., \& Bao, Y. 2004, New Astronomy, 9, 663

Zakharov, A. F., \& Repin, S. V. 1999, Astron. Rep., 43, 705

Zakharov, A. F., \& Repin, S. V. 2002a, Astron. Rep., 46, 360

Zakharov, A. F., \& Repin, S. V. 2002b, in Proc. of the Eleven Workshop on General Relativity and Gravitation in Japan, Waseda University, Tokyo, ed. J. Koga, T. Nakamura, K. Maeda, \& K. Tomita, 68

Zakharov, A. F., \& Repin, S. V. 2003a, A\&A, 406, 7

Zakharov, A. F., Repin, S. V. 2003b, in XEUS - studying the evolution of the hot Universe, ed. G. Hasinger, Th. Boller, \& A. N. Parmer, MPE Rep., 281, 339

Zakharov, A. F., \& Repin, S. V. 2003c, Astron. Rep., 47, 733

Zakharov, A. F., \& Repin, S. V. 2003d, Nuovo Cimento B, 118, 1193

Zakharov, A. F., \& Repin, S. V. 2004a, Adv. Space Res. 34, 1837

Zakharov, A. F., \& Repin, S. V. 2004b, in Frontiers of the Universe, ed. L. Celniker, \& J. Trân Thanh Vân, Proc. XIIIrd Rencontres de Blois (The GIOI Publishers), 229

Zakharov, A. F., \& Repin, S. V. 2005a, in Proc. of the 5th Serbian Conf. on Spectral Line Shapes in Astrophysics, ed. M. S. Dimitrijević, \& L. Č Popović, Mem. S. A. It., in press

Zakharov, A. F., \& Repin, S. V. 2005b, New Astron., submitted

Zakharov, A. F., Nucita, A. A., De Paolis, F., \& Ingrosso, G. 2005a, New Astron., 10, 479

Zakharov, A. F., Nucita, A. A., De Paolis, F., \& Ingrosso, G. 2005b, in Proc. 16th SIGRAV Conf. General Relativity and Gravitational Physics, ed. G. Vilasi, G. Esposito, G. Lambiase, G. Marmo, \& G. Scarpetta, AIP Conf. Proc., 751, 227

Zakharov, A. F., Nucita, A. A., De Paolis, F., \& Ingrosso, G. 2005c, in Proc. XXVII Workshop Fundamental Problems of High Energy and Field Theory, ed. V. A. Petrov, Institute for High Energy Physics, Protvino, 21, [arXiv: gr-qc/0507118]

Zakharov, A. F., Nucita, A. A., De Paolis, F., \& Ingrosso, G. 2005d, in Very High Energy Phenomena in the Universe, ed. J. Trân Thanh Vân, \& J. Dumarchez, Proc. XXXXth Rencontres de Moriond, in press

Zakharov, A. F., Nucita, A. A., De Paolis, F., \& Ingrosso, G. 2005e, in Proc. 22nd Texas Symp. Relativistic Astrophysics at Stanford University, ed. P. Chen, E. Bloom, G. Madejski, \& V. Petrosian, SLAC-R-752, eConf:C041213, http:// www. slac.stanford.edu/econf/CQ41213, Paper 1226

Zamir, R. 1993, ApJ, 403, 278 